\title{
Effects of nuclear symmetry energy on $\eta$ meson production and its rare decay to the dark U-boson in heavy-ion reactions
}

\author{
Gao-Chan Yong1, 田 and Bao-An Li ${ }^{2}$, 田 \\ ${ }^{1}$ Institute of Modern Physics, Chinese Academy of Sciences, Lanzhou 730000, China \\ ${ }^{2}$ Department of Physics and Astronomy, Texas A $8 M$ University-Commerce, Commerce, TX 75429, USA \\ and Department of Applied Physics, Xi'an Jiaotong University, Xi'an 710049, China
}

\begin{abstract}
Using a relativistic transport model ART1.0, we explore effects of nuclear symmetry energy on $\eta$ meson production and its rare decay to the dark U-boson in heavy-ion reactions from 0.2 to 10 $\mathrm{GeV} /$ nucleon available at several current and future facilities. The yield of $\eta$ mesons at sub-threshold energies is found to be very sensitive to the density dependence of nuclear symmetry energy. Above a beam energy of about $5 \mathrm{GeV} /$ nucleon in $\mathrm{Au}+\mathrm{Au}$ reactions, the sensitivity to symmetry energy disappears. Using the branching ratio of the rare $\eta$ decay $(\eta \rightarrow \gamma U)$ available in the literature, we estimate the maximum cross section for the U-boson production in the energy range considered, providing a useful reference for future U-boson search using heavy-ion reactions.
\end{abstract}

PACS numbers: 25.75.-q, 21.65.Mn, 21.65.Ef, 95.35.+d

\section{INTRODUCTION}

The physics motivations of this work are twofold. The first purpose is to look for new and possibly more sensitive probes of the high-density behavior of nuclear symmetry energy in high energy heavy-ion collisions. The other purpose is to explore the possibility of producing the neutral vector U-boson (dark photon) introduced in the super-symmetric extension of the Standard Model (SM) [1 5] in relativistic heavy-ion collisions. The two purposes naturally come together in studying properties of neutron stars where both the possible existence of the U-boson and the high-density behavior of nuclear symmetry energy affect significantly the Equation of State (EOS) of dense neutron-rich nucleonic matter. In fact, it has been shown already that massive neutron stars can be supported even with a rather soft EOS for symmetric nuclear matter and/or a super-soft symmetry energy if the additional interaction between two nucleons due to the exchange of a U-boson is considered [6, 7]. Moreover, both the symmetry energy and the possible U-boson affect the core-crust transition density/pressure and the moment of inertia of neutron stars [8].

A. Potentials of using $\eta$ meson production in heavy-ion collisions as a probe of the high-density behavior of nuclear symmetry energy

Nuclear symmetry energy $E_{\text {sym }}(\rho)$ encodes the energy cost of converting all protons into neutrons in symmetric nuclear matter. Because the density dependence of $E_{\text {sym }}(\rho)$ is very important for understanding properties of rare isotopes and neutron stars as well as the dynamics of heavy-ion reactions, supernova explosions

\footnotetext{
*Electronic address: yonggaochan@impcas.ac.cn
}

† Electronic address: Bao-An.Li@tamuc.edu and gravitation wave emissions of spiraling neutron star binaries, much efforts have been devoted to extracting the $E_{\text {sym }}(\rho)$ using data from both terrestrial laboratory experiments and astrophysical observations, see e.g., refs. [9 22]. While significant progress has been made recently in constraining the $E_{\text {sym }}\left(\rho_{0}\right)$ and the slope $L\left(\rho_{0}\right) \equiv\left[3 \rho\left(\partial E_{\mathrm{sym}} / \partial \rho\right]_{\rho_{0}}\right.$ at normal density $\rho_{0}$, see, e.g., refs. [23 49], our knowledge about the high-density behavior of the $E_{\text {sym }}(\rho)$ is still very poor. In fact, findings about the high-density $E_{\text {sym }}(\rho)$ from comparing heavyion reaction data with various transport model calculations are still inconsistent, see, e.g., refs. 50 53. This is at least partially because of both the uncertainties in the physics inputs and the different numerical techniques in initializing and modeling the transport process of colliding nuclei, see, e.g., refs. [53 58] for discussions on some of the contributing factors. Thus, new and possibly more sensitive probes of the high-density behavior of nuclear symmetry energy are always useful. Since the symmetry (isovector) potential is normally much smaller than the isoscalar potential, in order to observe clearly effects of the symmetry energy/potential in heavy-ion reactions one needs ideally to use slowly moving particles to be acted on by the isovector potential for a long time in a large region of high isospin asymmetry and density gradients. Sub-threshold mesons, such as pions and kaons, especially the ratios of different charge states, are good candidates and have been studied extensively. Transport model calculations have shown consistently that near their respective production threshold, the $\pi^{-} / \pi^{+}\left[\begin{array}{lll}59 & 63\end{array}\right.$ and $K^{+} / K^{0}$ [64] ratios are indeed sensitive to the highdensity symmetry energy although comparisons with limited data available are still inconclusive so far. Generally speaking, more massive mesons probe the $E_{\text {sym }}(\rho)$ at higher densities if effects of the final state interactions do not wash out the signal. The $\eta$ meson of mass 547.853 $\mathrm{MeV} / \mathrm{c}^{2}$ [65] is the most massive member in the octet of pseudoscalar Goldstone mesons. It has significant photon and dilepton decay channels providing the possibility of 
studying the high-density symmetry energy more cleanly using the electromagnetic probes. In fact, $\eta$ meson production in heavy-ion collisions has been studied extensively both theoretically and experimentally, especially by the TAPS and HADES Collaborations, see, e.g., refs. [66 75]. Moreover, as pointed out earlier [66, 67], because of the hidden strangeness (the $s \bar{s}$ component), $\eta$ mesons experience weaker final state interactions compared to pions. Contrary to kaons, because the net strangeness content is zero in $\eta$ mesons, they can be produced without another strange partner in the final state and thus require less energies. More quantitatively, the threshold invariant energy for $\eta$ and kaon production in nucleonnucleon collisions is $(\sqrt{s})_{t h}^{\eta}=2.4 \mathrm{GeV}$ and $(\sqrt{s})_{t h}^{K}=2.6$ $\mathrm{GeV}$, respectively. The corresponding threshold beam energy in free nucleon-nucleon collisions is $E_{t h}^{\eta}=1.2$ $\mathrm{GeV}$ and $E_{t h}^{K}=1.6 \mathrm{GeV}$, respectively. For a comparison, the experimental $K^{+} / \pi^{+}$and $\eta / \pi^{0}$ ratios in the most central $\mathrm{Au}+\mathrm{Au}$ reactions at $1 \mathrm{GeV} /$ nucleon is approximately $2 \times 10^{-3}\left[76\right.$ ] and $1.4 \times 10^{-2}$ [77], respectively. It is thus interesting to know if the $\eta$ meson might be useful for exploring the $E_{\text {sym }}(\rho)$. Intuitively, in order for the p-n pair to create a $\eta$ near its production threshold, they have to have their momenta oriented opposite to each other. Thus, near threshold $\eta$ mesons will probe sensitively the p-n relative momentum which is determined mainly by the gradient of the isovector potential. Compared to pions and kaons, however, the elementary $\eta$ production cross sections in baryon-baryon and mesonbaryon scattering still suffer from relatively larger uncertainties although they are gradually better known as more data and calculations become available 68,78 ]. It is thus necessary to know the optimal beam energies and the kinematic regions where the yield of $\eta$ mesons is most sensitive to the $E_{\text {sym }}(\rho)$.

\section{B. Heavy-ion reactions as a possible tool for dark U-boson search}

While there are well established observational evidences for dark matter through its gravitational interactions, the mass and interactions of dark matter particles are completely unknown [79]. Thus, what is dark matter? It is actually at the very top of the Eleven Science Questions for the New Century identified by the Committee on the Physics of the Universe, US National Research Council [80]. In fact, much efforts have been devoted to searching for dark matter candidates, see, e.g., refs. 81, 82 for reviews. Besides the weakly interacting massive particles as the most popular candidates for dark matter, it has been proposed that light dark matter particles $\chi$ with a mass in the range of 0.5 $20 \mathrm{MeV}$ may exist [1 5]. In particular, the annihilation of $\chi \bar{\chi} \rightarrow e^{+} e^{-}$through the exchange of the light vector U-boson of mass $10-100 \mathrm{MeV}$, has been used to explain successfully the INTEGRAL satellite observation of the excess flux of $511 \mathrm{keV}$ photons from the center re- gion of our galaxy [83] although alternative explanations exist 84]. Moreover, assuming the U-boson couples to quarks as well as electrons [79], the extra contribution to the pion decay $\left(\pi^{0} \rightarrow e^{+} e^{-}\right)$width mediated by an off-shell U-boson can explain nicely the enhanced pion decay observed by the KTeV Collaboration [85] in comparison with the Standard Model prediction [86]. Furthermore, as mentioned earlier, the extra interaction between nucleons due to the U-boson exchange may affect properties of neutron stars $[6-8,87]$ although it has no effect on properties of finite nuclei [88]. It may also cause the deviation from the inverse-square-law of gravity, see, e.g., [89 94] for recent reviews. The aforementioned studies and findings have motivated considerable interest in searching for the U-boson [95]. While various upper limits on the strength and interaction range of the U-boson have been put forward at various length scales without contradicting known physics principles and existing experimental/observational data, see, e.g., ref. [88] for the latest review, most of the constraints on properties of the U-boson are indirect. It is thus very interesting to see that some direct constraints on this kind of gauge bosons through neutral meson decays in neutrino experiments at CERN have been reported recently [96]. Moreover, several proposals to investigate directly properties of the U-boson in low energy $\left(E_{c . m} \leq 10 \mathrm{GeV}\right)$ experiments have been put forward. These include experiments using $e^{+} e^{-}$colliders through the $e^{+} e^{-} \rightarrow \gamma U$ process [4, 95, 97 99], meson rare decays at meson factories and fixed target electron-nucleus scatterings [95]. Many mesons can decay into the U-boson with branching ratio $B R(X \rightarrow Y+U) \approx \epsilon^{2} B R(X \rightarrow Y+\gamma)$ where $\epsilon$ is the strength ratio of the U-SM particle to $\gamma$-SM particle coupling. This is then followed by the $\mathrm{U}$ decay into dileptons, i.e., $U \rightarrow \ell^{+} \ell^{-}$. Using the meson summary tables of the Particle Data Group, Reece and Wang estimated the Uproducing branching ratios for several mesons [95]. The $\eta$ rare decay was shown to be the most promising one. More quantitatively, the ratio of $\eta \rightarrow \gamma U, \omega \rightarrow \pi^{0} U$, $\phi \rightarrow \eta U$ and $K_{L}^{0} \rightarrow \gamma U$ is found to be approximately $320 / 225 / 1.3 / 4.4 \times 10^{-3}$. It is interesting to note that the $\gamma+p \rightarrow \eta+p$ reaction with a cross section of approximately $70 \mathrm{nb}$ will be used for the U-boson search at the Jlab Eta Factory [100]. As we shall estimate in this work, the $\eta$ production cross section in a $\mathrm{Au}+\mathrm{Au}$ collisions from 0.2 to $10 \mathrm{GeV} / \mathrm{A}$ beam energy range is on the order of $60-6000 \mathrm{mb}$. While it will be very challenging to extract information about the U-boson from various backgrounds, the relatively large $\eta$ production cross section warrants a U-boson search using heavy-ion facilities, such as the CSR (Cooler Storage Ring) and the planned HIAF (High Current Heavy-Ion Accelerator Facility) in Lanzhou, the FAIR (Facility for Antiproton and Ion Research) in Darmstadt, NICA (Nuclotron based Ion Collider Facility) at JINR Dubna and the BES (Beam Energy Scan) program at RHIC (Relativistic Heavy-Ion Collider). To explore the idea more quantitatively, we shall investigate the excitation function of $\eta$ production 
in $\mathrm{Au}+\mathrm{Au}$ reactions in the energy range covered by these facilities.

\section{TRANSPORT MODEL SIMULATIONS OF $\eta$ MESON PRODUCTION IN HEAVY-ION COLLISIONS}

Our study is based on the relativistic transport model ART [101]. The model was developed from the BUU transport model [102] by including more baryon and meson resonances and their interactions. More specifically, the following baryons $N, \Delta(1232), N^{*}(1440), N^{*}(1535), \Lambda, \Sigma$, and mesons $\pi, \rho, \omega, \eta, K$ with their explicit isospin degrees of freedom are included. The model was used successfully in studying many features of heavy-ion reactions at AGS energies up to a beam momentum of about 15 $\mathrm{GeV} / \mathrm{c}$, for a review, see, ref. [103]. An extended version of ART is also used as a hadronic afterburner in the AMPT (A Multi-Phase Transport Model) model for heavy-ion collisions at RHIC and LHC energies 104. In the ART model, $\eta$ meson production and absorption are modeled via $\eta N \leftrightarrow N^{*}(1535)$. The cross sections for the production and absorption of $N^{*}(1535)$ resonance in baryon-baryon collisions and its decay width can be found in refs. 68, 101]. For ease of the following discussions, we emphasize that $\sigma\left(N N \rightarrow N N^{*}(1535)\right) \approx$ $2 \sigma(N N \rightarrow N N \eta)$ and the elementary cross section for $\eta$ production is strongly isospin dependent, i.e., $\sigma(p n \rightarrow$ $p n \eta) \approx 3 \sigma(p p \rightarrow p p \eta)=3 \sigma(n n \rightarrow n n \eta)$. The ART1.0 has the option of using different isoscalar potentials for baryons. However, the isovector (symmetry) potential was not implemented earlier. For this exploratory study, we use the Skyrme-type parametrization for the isospindependent but momentum-independent mean field potential [101, 105]

$$
\begin{aligned}
U(\rho, \delta, \tau) & \equiv A\left(\rho / \rho_{0}\right)+B\left(\rho / \rho_{0}\right)^{\sigma} \\
& +4 \tau E_{\mathrm{sym}}^{\mathrm{pot}}(\rho)+(18.6-F(x)) \\
& \times(G(x)-1)\left(\rho / \rho_{0}\right)^{G(x)} \delta^{2} .
\end{aligned}
$$

In the above, $E_{s y m}^{\text {pot }}(\rho)=F(x) \rho / \rho_{0}+(18.6-$ $\left.F(x))\left(\rho / \rho_{0}\right)^{G(x)}\right)$ is the interaction part of nuclear symmetry energy, $\delta \equiv\left(\rho_{n}-\rho_{p}\right) / \rho$ is the isospin asymmetry at density $\rho$ and $\tau=1 / 2(-1 / 2)$ for neutrons (protons). Adding the kinetic part of nuclear symmetry en$\operatorname{ergy} E_{\text {sym }}^{\text {kin }}(\rho)=\hbar^{2} / 6 m_{n}\left(3 \pi^{2} \rho / 2\right)^{2 / 3}$ where $m_{n}$ is the nucleon mass, the symmetry energy corresponding to the single-particle potential in Eq. 1 is

$$
\begin{aligned}
E_{\text {sym }}(\rho) & =E_{\text {sym }}^{\text {kin }}(\rho)+E_{\text {sym }}^{\text {pot }}(\rho) \\
& =\hbar^{2} / 6 m_{n}\left(3 \pi^{2} \rho / 2\right)^{2 / 3} \\
& +F(x) \rho / \rho_{0}+(18.6-F(x))\left(\rho / \rho_{0}\right)^{G(x)}
\end{aligned}
$$

with $F(x)$ and $G(x)$ given in Ref. [105] for different values of the parameter $\mathrm{x}$ used to vary the density dependence of
$E_{\text {sym }}$ [106]. Shown in Fig. 1 is the $E_{\text {sym }}(\rho)$ with $x=1,0$ and -2 , respectively, from being very soft to very stiff. The mean-field potentials of mesons and baryon resonances in nuclear matter are still largely unknown. We adopt here the minimum assumption that the isoscalar part of the mean-field potential for baryon resonances is the same as that for nucleons and there is no mean-field for mesons as assumed in most transport models. The isovector potential for baryon resonances is taken as an average of that for neutrons and protons with an isospindependent weighting factor determined by the square of the Clebsch-Gordan coefficients in the $\Delta\left(N^{*}\right) \leftrightarrow \pi+N$ coupling [59]. Contributions of the different charge states of baryon resonances to the isospin asymmetry of the baryonic system are similarly calculated [59]. We choose two sets of parameters $\mathrm{A}$ and $\mathrm{B}$, i.e., $\mathrm{A}=-356(-124)$ $\mathrm{MeV}, \mathrm{B}=303(70.5) \mathrm{MeV}$ and $\sigma=7 / 6(2)$ corresponding to the incompressibility of nuclear matter $\mathrm{K} \sim 200(380)$ $\mathrm{MeV}$ at $\rho_{0}=0.168 \mathrm{fm}^{-3}$.

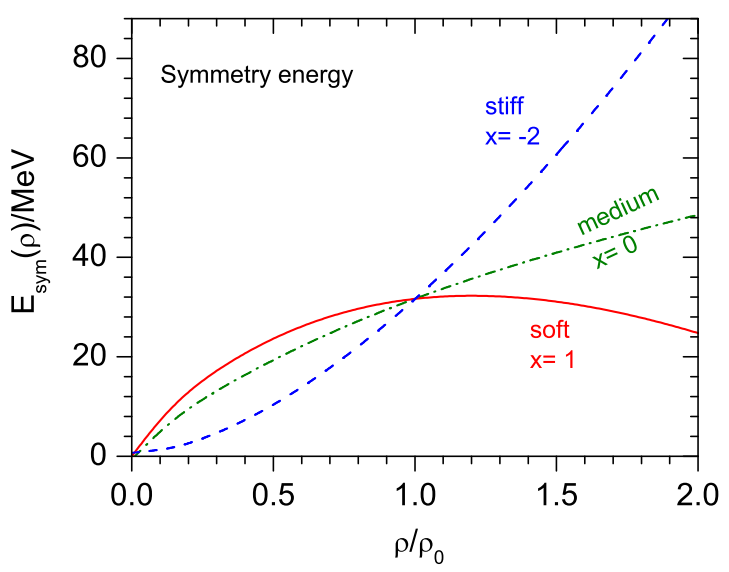

FIG. 1: (Color online) Nuclear symmetry energy as a function of reduced density $\rho / \rho_{0}$ (the symmetry energy at $\rho_{0}$ is taken to be $31.6 \mathrm{MeV}$ ) with three values for the parameter $x$. Taken from Ref. [105].

\section{EFFECTS OF NUCLEAR SYMMETRY ENERGY ON $\eta$ MESON PRODUCTION}

Shown in Fig. 2 is a comparison of the ART1.0 model calculations with the TAPS experimental data on the $\eta$ transverse momentum distribution at midrapidity in $\mathrm{Au}+\mathrm{Au}$ reaction at a beam energy of $1 \mathrm{GeV} /$ nucleon. In this and the following model calculations, the error bars are statistical in nature. It is seen that our calculations give a reasonable description of the data with $x=0$ and 1 while the stiff nuclear symmetry energy with $\mathrm{x}=-2$ leads to a significantly larger number of $\eta$ mesons. For a comparison, we notice that the FOPI $\pi^{-} / \pi^{+}$data in $\mathrm{Au}+\mathrm{Au}$ reactions at beam energies from about 0.4 


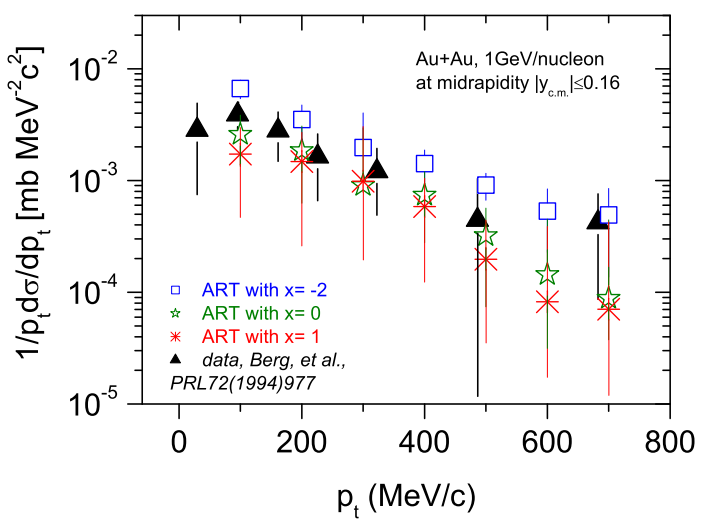

FIG. 2: (Color online) Transverse momentum distributions of midrapidity $\eta$ mesons in inclusive $\mathrm{Au}+\mathrm{Au}$ reaction at a beam energy of $1 \mathrm{GeV} /$ nucleon with different nuclear symmetry energies. Data are taken from Ref. [70].

to $1 \mathrm{GeV} /$ nucleon favor the symmetry energy with $\mathrm{x}=1$ [50]. Because $\eta$ is sensitive to the number of p-n collisions while the $\pi^{-} / \pi^{+}$ratio is determined by the ratio of n-n and p-p colliding pairs, $\eta$ and the $\pi^{-} / \pi^{+}$ratio provide complementary information about the symmetry energy. It has been argued that sub-threshold $\eta$ production requires multiple nucleon-nucleon scattering over sufficiently long time [66], it may thus carry information mainly about the equilibrium phase of the reaction. At this stage, the following condition is satisfied between any two regions of density (isospin asymmetry) $\rho_{1}\left(\delta_{1}\right)$ and $\rho_{2}\left(\delta_{2}\right)$ [107, 108]

$$
E_{\text {sym }}\left(\rho_{1}\right) \delta_{1}=E_{\text {sym }}\left(\rho_{2}\right) \delta_{2} \text {. }
$$

Then, the so-called isospin fractionation occurs, i.e., the density region with a higher symmetry energy $E_{\text {sym }}(\rho)$ will have a lower isospin asymmetry $\delta$ and vice versa. Consequently, with a stiffer symmetry energy the isospin asymmetry $\delta$ is lower in the high density regions where then more $n p(p n)$ collisions can occur to produce more $\eta$ mesons. While it is the opposite for the $\pi^{-} / \pi^{+}$ratio.

It is well known that the $\pi^{-} / \pi^{+}$ratio is more sensitive to the symmetry energy at lower beam energies especially in the sub-threshold region where the meanfield dominates the reaction dynamics and has longer time to modify the momentum of nucleons. To create a $\eta$ at sub-threshold energies requires even longer reaction time for both multiple collisions and the mean-field to act coherently in order to accumulate enough energy on the two colliding baryons or their resonances. Thus, it is interesting to examine the multiplicity of $\eta$ production as a function of incident beam energy. Shown in Fig. 3 are the $\eta$ multiplicity as a function of beam energy in inclusive $\mathrm{Au}+\mathrm{Au}$ reactions with both $x=0$ and $x=-2$. It is seen that the $\eta$ multiplicity decreases

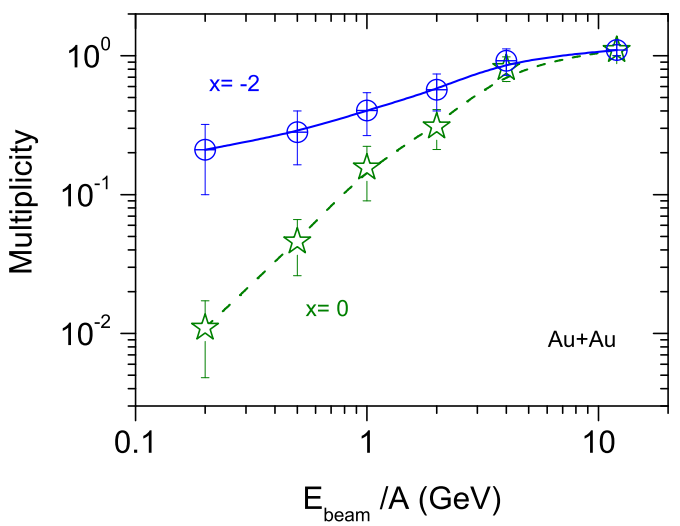

FIG. 3: (Color online) Multiplicity of inclusive $\eta$ production as a function of incident beam energy in $\mathrm{Au}+\mathrm{Au}$ reactions with the two different values for the symmetry energy parameter $\mathrm{x}$.

rapidly with decreasing beam energy, especially for the soft symmetry energy. The $\eta$ multiplicity saturates at an incident energy of about $10 \mathrm{GeV} /$ nucleon. It is interesting to see that indeed the effect of nuclear symmetry energy is stronger in the deeper sub-threshold region as one expects. In fact, it is more sensitive to the symmetry energy than the $\pi^{-} / \pi^{+}$ratio in the same energy region [109] although the pion yields are relatively more abundant. More detailed information about $\eta$ produc-

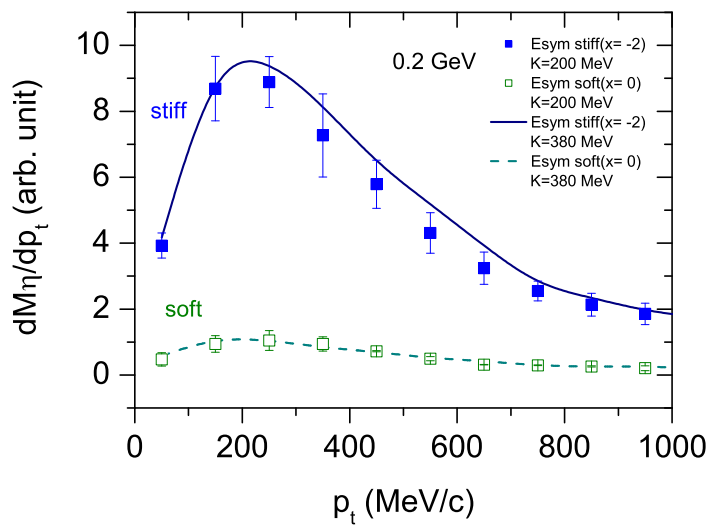

FIG. 4: (Color online) Transverse momentum distributions of $\eta$ production in inclusive $\mathrm{Au}+\mathrm{Au}$ reaction at incident beam energy of $0.2 \mathrm{GeV} /$ nucleon with different nuclear symmetry energies and compression coefficients of symmetric matter.

tion and its dependence on the symmetry energy can be obtained from studying its transverse momentum distri- 
bution. Shown in Fig. 4 is such a distribution in terms of $d M_{\eta} / d p_{t} \equiv \frac{d M_{\eta}\left(p_{t} \rightarrow p_{t}+d p_{t}\right)}{d p_{t}}$ for $\mathrm{Au}+\mathrm{Au}$ reactions at a beam energy of $0.2 \mathrm{GeV} /$ nucleon with different values for the parameter $\mathrm{x}$ and the incompressibility $K$. It is seen that at a transverse momentum of about $200 \sim 300$ $\mathrm{MeV} / \mathrm{c}$, the effect of nuclear symmetry energy reaches its maxima of about a factor of 5-10. Changing the incompressibility of nuclear matter from $\mathrm{K}=200 \mathrm{MeV}$ to $\mathrm{K}=380 \mathrm{MeV}$ seems to have little effect on the $\eta$ yields and its spectrum. While the larger (smaller) incompressibility of nuclear matter causes smaller (larger) compression during the reaction, both the production and reabsorption of $\eta$ are approximately equally affected. Moreover, as mentioned earlier, the p-n relative momentum is essentially determined by the gradient of the isovector potential with little influence from the isoscalar potential. Thus, the near-threshold $\eta$ production is sensitive to the symmetry energy but not the incompressibility of symmetric nuclear matter.

\section{PRODUCTION OF DARK U-BOSON FROM THE RARE DECAY OF $\eta$ MESONS IN HEAVY-ION COLLISIONS}

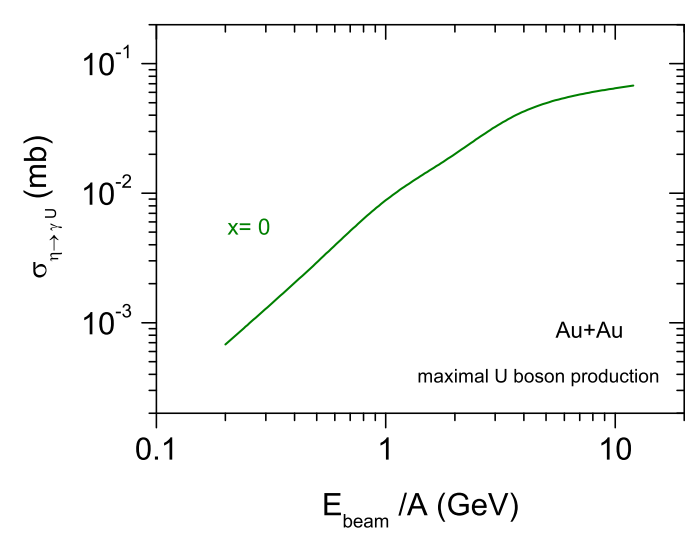

FIG. 5: (Color online) The maximal possible U-boson production cross section from the $\eta$ rare decay in inclusive $\mathrm{Au}+\mathrm{Au}$ reaction as a function of beam energy with the soft symmetry energy $(x=0)$.

We now turn to estimating the maximum U-boson production cross section. For this purpose, we use the maximal U-boson-SM particle coupling constant $\epsilon^{2}=$ 4. $\times 10^{-6}$ and $\left.B R(X \rightarrow Y+\gamma) \simeq 0.7[65,95]\right)$. Since the symmetry energy with $\mathrm{x}=0$ can better reproduce the $\eta$ production data from the TAPS collaboration, we present here our estimates using the soft symmetry energy with $\mathrm{x}=0$. Using the stiff symmetry energy with $\mathrm{x}=-2$ would increase the maximal total U-boson production cross section by the same ratio shown in Fig. 3. Shown in Fig. 5 is the maximal total U-boson production cross section in $\mathrm{Au}+\mathrm{Au}$ reaction at a beam energy from 0.2 to $10 \mathrm{GeV} /$ nucleon. It is seen that the maximum U-boson production cross section is in the 1-70 $\mu \mathrm{b}$ range which is about $6-8$ orders of magnitude higher than that estimated for the $\gamma+p$ reaction at the Jlab Eta Factory. However, similar to the situations at other facilities, it will be a big challenge to find in the dileption spectrum in heavy-ion collisions the signature of the dark U-boson decay $U \rightarrow \ell^{+} \ell^{-}$because of the various backgrounds. Moreover, the predicted U-boson width is only about $10 \mathrm{eV}$ which is much smaller than the resolution of most detectors. Nevertheless, our estimate of the excitation function of U-boson production in heavy-ion collisions provide a useful reference for future U-boson search.

\section{CONCLUSIONS}

In summary, within the transport model ART1.0 we studied the excitation function of $\eta$ yield in heavy-ion collisions both as a potential probe of the symmetry energy and as a possible source of the dark U-boson. The yield of $\eta$ meson is found to be very sensitive to the density dependence of nuclear symmetry energy at subthreshold energies. Using the branching ratio of the rare $\eta$ decay $(\eta \rightarrow \gamma U)$ available in the literature, we estimated the maximum cross section for the U-boson production in heavy-ion collisions at a beam energy of 0.2 to $10 \mathrm{GeV} /$ nucleon, providing a useful reference for future U-boson search experimentally.

\section{ACKNOWLEDGEMENTS}

This work was supported in part by the National Natural Science Foundation of China under Grant No. 11175219, the IMP principal fund, the U.S. National Science Foundation grants PHY-0757839 and PHY-1068022, the U.S. National Aeronautics and Space Administration under grant NNX11AC41G issued through the Science Mission Directorate, and through CUSTIPEN (ChinaU.S. Theory Institute for Physics with Exotic Nuclei) under U.S. DOE grant number DE-FG02-13ER42025.
[1] C. Boehm et al., Phys. Rev. Lett. 92 (2004) 101301.

[2] C. Boehm and P. Fayet, Nucl. Phys. B683 (2004) 219.
[3] Pierre Fayet, Phys. Rev. D74 (2006) 054034.

[4] Natalia Borodatchenkova, Debajyoti Choudhury, and 
Manuel Drees, Phys. Rev. Lett. 96 (2006) 141802.

[5] P. Fayet, Phys. Lett. B675 (2009) 267.

[6] M.I. Krivoruchenko et al., Phys. Rev. D79 (2009) 125023.

[7] De-Hua Wen, Bao-An Li, Lie-Wen Chen, Phys. Rev. Lett. 103 (2009) 211102.

[8] Hao Zheng and Lie-Wen Chen, Phys. Rev. D85 (2012) 043013.

[9] B.A. Li, C.M. Ko, W. Bauer, Topical Review, Int. J. Mod. Phys. E 7 (1998) 147.

[10] B.A. Brown, Phys. Rev. Lett. 85 (2000) 5296.

[11] B. A. Li and W. Udo Schröder, Isospin Physics in Heavy-Ion Collisions at Intermediate Energies, Nova Science Publishers, Inc, New York, 2001.

[12] J.M. Lattimer, M. Prakash, Science 304 (2004) 536.

[13] A.W. Steiner, M. Prakash, J.M. Lattimer, P.J. Ellis, Phys. Rep. 411 (2005) 325.

[14] V. Baran, M. Colonna, V. Greco, M. Di Toro, Phys. Rep. 410 (2005) 335.

[15] B.A. Li, L.W. Chen and C.M. Ko, Phys. Rep. 464 (2008) 113.

[16] EOS working group report, https://groups.nscl.msu.edu/hira/EoS.htm

[17] W.G. Newton, M. Gearheart, D.H. Wen, B.A. Li, J. of Phys.: Conference Series 420,(2013) 012145.

[18] L.W. Chen, arXiv:1212.0284, World Scientific (2013) in press.

[19] B.A. Li, L.W. Chen, F.J. Fattoyev, W.G. Newton and C. Xu, J. of Phys.: Conference Series, 413 (2013) 012021.

[20] J. Lattimer, Annu. Rev. Nucl. Part. Sci. 62 (2012) 485.

[21] M.B. Tsang et al., Phys. Rev. C 86 (2012) 024605.

[22] F. J. Fattoyev, J. Carvajal, W. G. Newton and B.A. Li, Phys. Rev. C87 (2013) 015806.

[23] L.W. Chen, Phys. Rev. C 83 (2011) 044308.

[24] P. Moller, W.D. Myers, H. Sagawa and S. Yoshida, Phys. Rev. Lett. 108 (2012) 052501.

[25] P. Danielewicz and J. Lee, Nucl. Phys. A 818 (2009) 36 .

[26] B.K. Agrawal, J.N. De and S.K. Samaddar, Phys. Rev. Lett. 109 (2012) 262501.

[27] Z.Y. Sun et al., Phys. Rev. C 82 (2010) 051603(R).

[28] M.B. Tsang et al., Phys. Rev. Lett. 92 (2004) 062701.

[29] M.B. Tsang et al.,Phys. Rev. Lett. 102 (2009) 122701.

[30] L.W. Chen, C.M. Ko and B.A. Li, Phys. Rev. Lett. 94 (2005) 032701.

[31] B.A. Li and L.W. Chen, Phys. Rev. C 72 (2005) 064611.

[32] D.V. Shetty, S.J. Yennello and G.A. Souliotis, Phys. Rev. C 76 (2007) 024606.

[33] J.M. Lattimer and Y. Lim, arXiv:1203.4286

[34] M. Centelles, X. Roca-Maza, X. Vinas and M. Warda, Phys. Rev. Lett. 102 (2009) 122502.

[35] M. Warda, X. Vinas, X. Roca-Maza and M. Centelles, Phys. Rev. C 80 (2009) 024316.

[36] L.W. Chen, C.M. Ko, B. A Li and J. Xu, Phys. Rev. C 82 (2010) 024321.

[37] M. Liu, N. Wang, Z. Li and F. Zhang, Phys. Rev. C 82 (2010) 064306.

[38] C. Xu, B.A. Li and L.W. Chen, Phys. Rev. C 82 (2010) 054607.

[39] A. Klimkiewicz et al., Phys. Rev. C 76 0(2007) $51603(\mathrm{R})$.

[40] A. Carbone et al., Phys. Rev. C 81 (2010) 041301(R).

[41] W.D. Myers and W.J. Swiatecki, Nucl. Phys. A 601
(1996) 141.

[42] Z. Kohley et al., Phys. Rev. C 82 (2010) 064601.

[43] I. Vidaña, Phys. Rev. C 85 (2012) 045808.

[44] D.H. Wen, W.G. Newton and B.A. Li, Phys. Rev. C 85 (2012) 025801.

[45] A.W. Steiner, J.M. Lattimer, and E.F. Brown, Astrophys. J. 722 (2010) 33.

[46] A.W. Steiner S. Gandolfi, Phys. Rev. Lett. 108 (2012) 081102.

[47] M. Gearheart, W.G. Newton, J. Hooker and B.A. Li, Mon. Not. Roy. Astron. Soc., 418 (2011) 2343.

[48] H. Sotani, K. Nakazato, K. Iida, K. Oyamatsu, Mon. Not. Roy. Astron. Soc. 428 (2013) L21.

[49] W.G. Newton and B.A. Li, Phys. Rev. C 80 (2009) 065809 .

[50] Z.G. Xiao, B.A. Li, L.W. Chen, G.C. Yong, M. Zhang, Phys. Rev. Lett. 102 (2009) 062502.

[51] Z.Q. Feng, G.M. Jin, Phys. Lett. B683 (2010) 140.

[52] P. Russotto, P.Z. Wu, M. Zoric, M. Chartier, Y. Leifels, R.C. Lemmon, Q. Li, J. Lukasik, A. Pagano, P. Pawlowski, W. Trautmann, Phys. Lett. B697 (2011) 471.

[53] M.D. Cozma, Phys. Lett. B700 (2011) 139.

[54] Yingxun Zhang, P. Danielewicz, M. Famianoe, Zhuxia Li, W.G. Lyncha, M.B. Tsang, Phys. Lett. B664 (2008) 145.

[55] D.D.S. Coupland, W.G. Lynch, M.B. Tsang, P. Danielewicz, Yingxun Zhang, Phys. Rev. C84 (2011) 054603.

[56] G.C. Yong, Eur. Phys. J. A46 (2010) 399.

[57] Y. Gao, L. Zhang, H.F. Zhang, X.M. Chen, G.C. Yong, Phys. Rev. C83 047602 (2011); Yingxun Zhang, D.D.S. Coupland, P. Danielewicz, Zhuxia Li, Hang Liu, Fei Lu, W. G. Lynch, and M. B. Tsang, Phys. Rev. C85 (2012) 024602.

[58] G.C. Yong, Y. Gao, W. Zuo, X.C. Zhang, Phys. Rev. C84 (2011) 034609.

[59] B.A. Li, Phys. Rev. Lett. 88 (2002) 192701; Nucl. Phys. A708 (2002) 365.

[60] T. Gaitanos, M. Di Toro, S. Typel, V. Baran, C. Fuchs, V. Greco, H.H. Wolter, Nucl. Phys. A 732 (2004) 24.

[61] B.A. Li, G.C. Yong, W. Zuo, Phys. Rev. C 71 (2005) 014608.

[62] G.Ferini, M.Colonna, T.Gaitanos, M.Di Toro, Nucl. Phys. A762 (2005) 147.

[63] Ming Zhang, et al., Phys. Rev. C80 (2009) 034616.

[64] G. Ferini, M. Colonna, T. Gaitanos, M. Di Toro, H. Wolter, Phys. Rev. Lett. 97 (2006) 202301; M. Di Toro, et al., Nucl. Phys. A 782 (2007) 267.

[65] J. Beringer et al. (Particle Data Group), Phys. Rev. D86 (2012) 010001.

[66] A. Depaoli, K. Niita, W. Cassing, U. Mosel and C.M. Ko, Phys. Lett. B219 (1989) 194.

[67] U. Mosel, Ann. Rev. Nucl. Part. Sci. 41 (1991) 29.

[68] Gy. Wolf, W. Cassing and U. Mosel, Nucl. Phys. A552 (1993) 549.

[69] V. Metag, Prog. Part. Nucl. Phys., 30 (1993) 75.

[70] F.D. Berg, et al. (TAPS Collaboration), Phys. Rev. Lett. 72 (1994) 977.

[71] A. Taranenko et al. (TAPS Collaboration), Czech. J. Phys. 50S4 (2000) 139.

[72] W. Cassing, E. Bratkovskaya, A. Sibirtsev, Nucl. Phys. A691 (2001) 745.

[73] G. Agakishiev et al. (HADES Collaboration), Phys. 
Lett. B 690 (2010) 118.

[74] G. Agakishiev et al. (HADES Collaboration), Phys. Rev. C 85 (2012) 054005.

[75] P. Salabura for the HADES Collaboration, J. of Physics: Conference Series 420, (2013) 012013.

[76] P. Senger, Acta Phys.Polon. B31 (2000) 2313-2320; Nucl.Phys. A685 (2001) 312-327.

[77] R. Averbeck, R. Holzmann, V. Metag and R.S. Simon, Phys. Rev. C67 (2003) 024903.

[78] M. Thomere, C. Hartnack, G. Wolf and J. Aichelin, Phys. Rev. C75 (2007) 064902.

[79] Y. Kahn, M. Schmitt, and T.M.P. Tait, Phys. Rev. D78 (2008) 115002.

[80] Connecting Quarks with the Cosmos:Eleven Science Questions for the New Century, National Research Council, The National academies Press, ISBN-10:0-30907406-1.

[81] Gerard Jungman, Marc Kamionkowski, Kim Griest, Phys. Rep. 267 (1996) 195.

[82] Gianfranco Bertone, Dan Hooper, Joseph Silk, Phys. Rep. 405 (2005) 279.

[83] P. Jean et al., Astron. Astrophys. 407 (2003) L55; J. Knodlseder et al., Astron. Astrophys. 411 (2003) L457.

[84] Georg Weidenspointner et al., Nature 451 (2008) 159.

[85] E. Ascasibar et al. (KTeV Collaboration), Phys. Rev. D75 (2007) 114007.

[86] A. E. Dorokhov and M. A. Ivanov, Phys. Rev. D75 (2007) 114007.

[87] Dong-Rui Zhang, Ping-Liang Yin, Wei Wang, Qi-Chao Wang, Wei-Zhou Jiang, Phys. Rev. C83 (2011) 035801.

[88] Jun Xu, Bao-An Li, Lie-Wen Chen and Hao Zheng, Journal of Physics G. 40 (2013) 035107.

[89] Adelberger, E. G., et al., 2003, Annu. Rev. Nucl. Part. Sci., 53, 77; Prog. Part. Nucl. Phys., 62 (2009) 102.

[90] E. Fischbach and C.L. Talmadge, The Search for NonNewtonian Gravity, Springer-Verlag, New York, Inc.
(1999), ISBN 0-387-98490-9.

[91] Serge Reynaud et al., Int. J. Mod. Phys. A20 (2005) 2294.

[92] R.D. Newman, E.C. Berg and P.E. Boynton, Space Science Review, 148 (2009) 175.

[93] J.P. Uzan, Rev. Mod. Phys., 75 (2003) 403.

[94] Slava G. Turyshev et al., arXiv:0902.3004 Astro2010 Science White Paper.

[95] Matthew Reece, Lian-Tao Wang, JHEP 0907 (2009) 051.

[96] S.N. Gninenko, Phys. Rev. D85 (2012) 055027; Phys. Lett. B713 (2012) 244.

[97] L. Barzè, et al., Acta Physica Polonica B42 (2011) 2461.

[98] Shou-hua Zhu, Phys. Rev. D75 (2007) 115004.

[99] H.B. Li, L. Tao, Phys. Lett. B686 (2010) 249.

[100] Liping Gan, Eta Rare Decay Proposal to Jlab 2012, http://wwwold.jlab.org/exp_prog/proposals/12/PR12-12-003.p

[101] B.A. Li and C.M. Ko, Phys. Rev. C52 (1995) 2037.

[102] G.F. Bertsch and S. Das Gupta, Phys. Rep., 160 (1988) 189.

[103] B.A. Li, C.M. Ko, A.T. Sustich and B. Zhang, Topical Review, Int. Journal of Modern Physics, Vol. 10, Nos. 4 \& 5 (2001) 267-352.

[104] Zi-Wei Lin, Che Ming Ko, Bao-An Li, Bin Zhang, Subrata Pal, Phys. Rev. C72 (2005) 064901.

[105] L.W. Chen, C.M. Ko and B.A. Li, Phys. Rev. Lett. 94 (2005) 032701.

[106] B.A. Li, C.B. Das, S. Das Gupta and C. Gale, Phys. Rev. C69 (2004) 011603; Nucl. Phys. A735 (2004) 563.

[107] L. Shi and P. Danielewicz, Europhys. Lett. 49 (2000) 34.

[108] B.A. Li, Nucl. Phys. A708 (2002) 365.

[109] F. Zhang, Y. Liu, G.C. Yong, W. Zuo, Chin. Phys. Lett. 29 (2012) 052502. 